\title{
Surgical Morbidity of Simultaneous Kidney and Pancreas Transplantation: A Single-Centre Experience in the Tacrolimus Era
}

\author{
Stephen E. Thwaites, ${ }^{1}$ Vincent W. T. Lam,, ${ }^{1,2}$ Jinna Yao,, ${ }^{1,2}$ Kathy Kable, ${ }^{1}$ \\ Lillian Jenkins, ${ }^{1}$ Cheng Chen, ${ }^{1}$ Paul Robertson, ${ }^{1}$ Wayne J. Hawthorne,, ${ }^{1,2}$ Brendan J. Ryan, ${ }^{1}$ \\ Henry C. Pleass, ${ }^{1,2}$ and Richard D. M. Allen ${ }^{1,2}$ \\ ${ }^{1}$ National Pancreas Transplant Unit, Westmead Hospital, Westmead, NSW 2145, Australia \\ ${ }^{2}$ Discipline of Surgery, Sydney Medical School, The University of Sydney, Sydney, NSW 2006, Australia \\ Correspondence should be addressed to Vincent W. T. Lam; vincent.lam@sydney.edu.au
}

Received 13 July 2012; Accepted 2 August 2012

Academic Editors: A. D. Hess and A. Rydzewski

Copyright (C) 2013 Stephen E. Thwaites et al. This is an open access article distributed under the Creative Commons Attribution License, which permits unrestricted use, distribution, and reproduction in any medium, provided the original work is properly cited.

Introduction. Simultaneous pancreas and kidney (SPK) transplantation is performed to restore normoglycaemia and renal function in patients with Type I diabetes mellitus and end-stage renal failure. We aimed to evaluate the impact of major postoperative complications to patient and graft survival outcomes. Method. Using a prospectively collected database over a 10-year period, major postoperative complications requiring return to operating theatre as well as patient and graft survival outcomes were analysed retrospectively. Results. Between January 2001 and May 2010, 165 patients underwent first-time SPK transplantation. Median age of recipients was 39.8 years (range, 16.9-53.2). Enteric drainage was used in 149 patients, and bladder drainage was used in 16. Median follow-up time was 5.2 years (range 1.1-10.3). Fifty-six patients (34\%) returned to operating theatre at least once. Pancreatic allograft loss secondary to vascular thrombosis occurred in 12 patients (7\%), and 2 patients (1.2\%) required transplant pancreatectomy due to debilitating pancreatic enzyme leaks. At 1 and 5 years, patient survival was $98 \%$ and $94 \%$; pancreas graft survival, $86 \%$ and $77 \%$; kidney graft survival $96 \%$ and $89 \%$, respectively. Conclusion. SPK is a safe and effective treatment for Type I diabetes mellitus and end-stage renal failure although surgical reintervention is required in approximately one-third of patients. Preventing vascular thrombosis remains a major challenge.

\section{Introduction}

The first successful simultaneous pancreas-kidney (SPK) transplantation was performed at the University of Minnesota Hospital in 1966 [1]. Since then, SPK has become an effective treatment modality for patients with Type 1 diabetes mellitus (T1DM) and end-stage renal failure [2].

Despite numerous advances in surgical techniques, organ preservation, and immunosuppression over the past 25 years, SPK is still associated with significant morbidity and rate of transplant graft loss remains significant [3]. Surgical complications in particular graft thrombosis and haemorrhage can lead to reintervention, graft loss, and increased length of hospital stay [4].
Our aim was to review the experience of SPK transplantation at the National Pancreas Transplant Unit (NPTU) in Australia over the last 10 years, focusing on the incidence of major postoperative complications and the reasons for graft loss. We also aimed to identify risk factors leading to graft loss within our cohort.

\section{Patients and Methods}

A retrospective study was conducted on 165 first-time SPK transplants performed at the NPTU between January 2001 and May 2010. This period was chosen because tacrolimusbased immunosuppression and enteric drainage of pancreas 
transplant graft became standard therapy in our unit in January 2001. Eligibility criteria for SPK transplantation included the following: T1DM, significant renal impairment, and age less than 50 years. Exclusion criteria included a body mass index (BMI) greater than 30, cigarette smoking, and significant untreated coronary artery disease.

Prior to being accepted onto the waiting list for transplantation, all patients were reviewed by a transplant physician and surgeon. Preoperative assessment included clinical evaluation, comprehensive biochemical studies, and cardiac stress tests.

\subsection{Organ Procurement, Preservation, and Transplantation.} With the exception of one donation after cardiac death (DCD), organs were recovered from heart-beating donors with certified brain death. The donor pancreas procurement procedure has been previously described [5]. In brief, the donor pancreas, duodenum, and spleen were recovered enbloc, and the root of the small bowel mesentery was stapled. Following 25,000 units of intravenous heparin and aortic flush with several litres of cold $\left(4^{\circ} \mathrm{C}\right)$ University of Wisconsin solution (UW), the organs were removed and placed in ice saline slush. The spleen was separated from the pancreas during back-table preparation. The donor duodenum was stapled proximally and distally and oversewn with 3-0 monofilament absorbable suture. The donor common iliac artery, including its bifurcation, was anastomosed as a Y-graft to the donor splenic artery and superior mesenteric artery. The donor common iliac vein was used as an extension to the donor portal vein.

In the recipient, iliac artery exposure was performed through bilateral Rutherford-Morrison incisions or a midline laparotomy. The kidney graft was anastomosed to the recipient's left iliac vessels. An external ureteroneocystostomy was performed over a 7-French ureteric stent. The donor pancreas was positioned in the right iliac fossa in an intraperitoneal position. The pancreatic head was placed inferiorly, and the donor vessels were anastomosed to the recipient's right iliac vessels.

Since 2001, enteric drainage has been the preferred route of exocrine drainage in our unit. To achieve this, the donor duodenum segment and recipient jejunum were anastomosed in a side-to-side configuration. In most cases, this was fashioned in two layers using a 3-0 monofilament absorbable suture although a small number were performed with linear cutting stapler [6]. For bladder-drained pancreases, the donor duodenum segment was anastomosed to the dome of the urinary bladder using 3-0 monofilament absorbable suture.

\subsection{Immunosuppression and Medical Therapy. Maintenance} immunosuppression typically consisted of a tacrolimus, mycophenolate mofetil and prednisolone. Induction therapy with monoclonal anti-CD25 antibody was used routinely after 2006.

Recipients received broad-spectrum antibiotic prophylaxis for 3 days, antifungal prophylaxis for one week, and
CMV prophylaxis for six months postoperatively. Subcutaneous heparin (5000 units three times daily) was administered during convalescence in hospital, and acetylsalicylic acid (100 mg daily) was commenced upon resumption of diet.

2.3. Statistical Analysis. Pancreas graft failure was defined as the day upon which permanent insulin administration was commenced or patient death. Kidney graft failure was defined as the day upon which permanent dialysis was commenced or death.

Measured values are reported as percentages for categorical data, and medians $+/$ - interquartile range (IQR; 25 th to 75 th quartile) for continuous data. Differences between groups were compared by the Chi-squared or Fisher's exact tests for categoric variables, Student's $t$ test for continuous parametric variables, and the Wilcoxon test for nonparametric continuous variables. Patient and graft survival was analysed by the Kaplan-Meier method. Logistic regression and Cox regression were used to assess risk factors for organ loss. Statistical analysis was performed using NCSS (Kaysville, Utah, USA) [7]. A value of $P<0.05$ was considered significant.

\section{Results}

3.1. Demographics. Between January 2001 and May 2010, 165 patients underwent SPK transplantation. Patient demographics are presented in Table 1. Despite entry criteria onto the transplant program, 11 patients had a BMI greater than 30 and six patients were aged 50 years or older at the time of transplantation. Most patients had been dialysis-dependent for at least one year, while $7 \%$ of patients had not progressed to dialysis at the time of transplantation. Sixteen patients (10\%) had peripheral vascular disease requiring amputations, 13 had minor (toe) amputations, and 3 had below-knee amputations. Nine of 18 patients with a history of coronary artery disease underwent revascularisation procedures prior to transplantation.

Organs were retrieved from around Australia, including 83 from New South Wales and the ACT, 23 from Queensland, 18 from Victoria, and 41 from Western Australia and South Australia combined. The longest pancreas cold ischaemia time (CIT) was 19.3 hours.

3.2. Surgical Complications and Return to Theatre. Eightyfive secondary operations were performed on 56 out of 165 recipients (34\%; Table 2). Seven patients had two or more distinct surgical complications. Twenty patients underwent more than one reoperation. Ten of the 20 patients $(50 \%)$ underwent drainage of intraabdominal collections (including those secondary to a pancreatic leak) on at least one occasion. Seven of the 20 (35\%) resumed insulin administration within 6 months of transplantation.

Thirty-six patients $(21.8 \%)$ returned to theatre within the first 30 days. Within this group, thrombosis was the most common reason for return to theatre $(n=11 ; 6.7 \%)$, followed by pancreatic leak $(n=7,4.2 \%$; Table 2$)$. In only one case of thrombosis was the pancreas graft able to be salvaged. One 
TABLE 1: Recipient and donor demographics.

\begin{tabular}{|c|c|}
\hline & $n=165(\%)$ \\
\hline \multicolumn{2}{|l|}{ Recipient } \\
\hline Median age, yr (range) & $39(16-53)$ \\
\hline Males & $87(53 \%)$ \\
\hline Median BMI (range) & $24.1(17.3-37.3)$ \\
\hline Duration of diabetes, yr (range) & $26(4-45)$ \\
\hline \multicolumn{2}{|l|}{ Duration of dialysis } \\
\hline Preemptive & $39(24 \%)$ \\
\hline $0-1$ year & $30(18 \%)$ \\
\hline $1-3$ years & $61(37 \%)$ \\
\hline$>3$ years & $35(21 \%)$ \\
\hline Prior renal transplant, failed & $2(1.2 \%)$ \\
\hline Coronary artery disease & $18(11 \%)$ \\
\hline Ex-smoker & $61(37 \%)$ \\
\hline \multicolumn{2}{|l|}{ Donor } \\
\hline Median donor age (years, IQR) & $26(20-36)$ \\
\hline \multicolumn{2}{|l|}{ Cause of death } \\
\hline Trauma & $111(67 \%)$ \\
\hline Nontraumatic causes & $54(33 \%)$ \\
\hline \multicolumn{2}{|l|}{ Organ } \\
\hline $\begin{array}{l}\text { Median pancreas cold ischaemic time } \pm \mathrm{SD} \text {, } \\
\mathrm{hr} \text { (range) }\end{array}$ & $12.1(10.2-14.2)$ \\
\hline $\begin{array}{l}\text { Median pancreas anastomotic time, } \\
\min (\mathrm{IQR})\end{array}$ & $27(24-31)$ \\
\hline $\begin{array}{l}\text { Median kidney cold ischaemic time } \pm \mathrm{SD} \text {, } \\
\mathrm{hr} \text { (range) }\end{array}$ & $10.8(9.2-13.2)$ \\
\hline Median kidney anastomotic time, $\min (\mathrm{IQR})$ & $30(25-38.5)$ \\
\hline
\end{tabular}

patient returned to theatre twice in this period-initially for postoperative haemorrhage and then for graft thrombosis.

After the first 30 days, pancreatic leak was the commonest cause of return to theatre $(n=7 ; 4.2 \%)$, followed by small bowel obstruction $(n=6 ; 3.6 \%)$.

In total, $15 / 165$ patients $(9.1 \%)$ had confirmed pancreatic leaks. One, which fistulated through the wound, was managed nonoperatively. Fourteen of the 15 patients with pancreatic leaks returned to theatre for a total of 26 reoperations (range 1-6); in eleven the exocrine drainage was revised, and in two $(1.2 \%)$ the allograft was removed due to ongoing sepsis.

While $11 / 15$ pancreatic leaks occurred within the first 30 days, in three cases the first manifestation of leak occurred more than six months postoperatively (at 7 months, 13 months, and 18 months). Despite extensive investigation at the time, no cause for the late leaks was established.

Leak occurred in 3/16 bladder-drained and 12/149 enterically drained pancreases $(P=0.16)$. Donor age $(P=0.67)$, degree of HLA-mismatch $(P=0.48)$, donor CMV status $(P=$ $0.06)$, the degree of pancreatic enzyme rise in the immediate post-operative period $(P=0.59)$, and the requirement for
TABLE 2: Return to theatre.

\begin{tabular}{|c|c|c|c|}
\hline \multirow{2}{*}{$\begin{array}{l}\text { Complication requiring } \\
\text { surgery }\end{array}$} & \multicolumn{3}{|c|}{$\begin{array}{l}\text { Presentation to theatre with de novo } \\
\text { condition }\end{array}$} \\
\hline & $<30$ days & 30-365 days & $>365$ days \\
\hline Pancreatic Thrombosis & 11 & $1^{\mathrm{a}}$ & 1 \\
\hline $\begin{array}{l}\text { Severe rejection with } \\
\text { secondary pancreatic } \\
\text { thrombosis }\end{array}$ & 2 & & 2 \\
\hline Pancreatic leak & 7 & 5 & 2 \\
\hline Haemorrhage & 4 & $1^{\mathrm{b}}$ & \\
\hline Abscess, lymphocoele & 4 & 4 & 1 \\
\hline $\begin{array}{l}\text { Exploratory laparotomy } \\
\text { (including for pancreatitis) }\end{array}$ & 3 & & 1 \\
\hline Ureteric & 3 & 1 & \\
\hline Small bowel obstruction & 2 & 3 & 3 \\
\hline Acute limb ischaemia & 1 & & \\
\hline Wound resuture & & 2 & \\
\hline Hernia & & 2 & \\
\hline Total & 37 & 19 & 10 \\
\hline
\end{tabular}

${ }^{\mathrm{a}}$ In the setting of CMV pancreatitis.

${ }^{\mathrm{b}}$ Following transplant renal biopsy.

parenteral immunosuppressive therapy (beyond the induction period) within the first year of transplantation $(P=0.36)$ were not associated with pancreatic leak.

3.3. Pancreas and Patient Survival. There were fifteen deaths from 165 recipients during a median followup of 5.2 years. The most common confirmed cause of death was opportunistic infection $(n=4)$, which included cryptococcal meningitis and fungal pneumonia. This was followed by malignancy $(n=3)$.

At 1 and 5 years, patient survival was $98 \%$ and $94 \%$, respectively. Kidney allograft survival at one and five years was $96 \%$ and $89 \%$, while pancreas allograft survival was $86 \%$ and $77 \%$, respectively (Figure 1 ).

Forty of 165 pancreas grafts (24\%) were lost over the 10 year period. The most common cause of pancreas graft loss was organ failure, including failure due to rejection $(n=$ 13, 7.9\%; Table 3). The most common cause of late graft loss was death with a functioning graft $(n=12,7.3 \%)$. In fact, recipient death accounted for $30 \%$ of all pancreas grafts lost. Acute rejection coincided with graft thrombosis in three patients; two within the first 30 days, and one at 20 months.

Of the 40 pancreas grafts lost, $23(58 \%)$ were lost in the first year, including two due to recipient death (Table 4). Fourteen of the $40(61 \%)$ were lost in the first month, the most common cause of which was thrombosis without evidence of rejection $(n=11)$.

Duration of dialysis greater than one year before SPK transplantation was associated with pancreas graft loss at one year $(P=0.03)$ and poorer long-term pancreas $(\mathrm{RR}=2.3$, 95\% CI 1.1-4.8), but not kidney ( $\mathrm{RR}=2.5,95 \%$ CI 0.9-6.7), graft survival. 
TABLE 3: Causes of pancreas loss.

\begin{tabular}{lccc}
\hline & $<1$ year & $>1$ year & Total \\
\hline $\begin{array}{l}\text { Thrombosis } \\
\text { Failure (including acute } \\
\text { and chronic rejection and } \\
\text { thrombosis secondary to } \\
\text { rejection) }\end{array}$ & 11 & 1 & $12(30 \%)$ \\
$\begin{array}{l}\text { Pancreatic leak } \\
\begin{array}{l}\text { Death with functioning } \\
\text { graft }\end{array}\end{array}$ & 2 & 6 & $13(32.5 \%)$ \\
Primary nonfunction & 1 & 0 & $2(5 \%)$ \\
\hline Total & 23 & 10 & $12(30 \%)$ \\
\hline
\end{tabular}

There was no significant difference in pancreas CIT between grafts that failed and grafts that were still functioning at one year $(P=0.06)$. The degree of serum lipase elevation within the first 24 hours of transplantation was also not associated with early graft loss $(P=0.41)$.

Reoperation correlated with earlier graft loss $(\mathrm{RR}=3.6$, 95\% CI $=1.8-6.8)$, including death-censored graft loss $(P<$ $0.001)$, but was not detrimental to patient survival $(P=0.72)$.

\section{Discussion}

Whole-organ pancreas transplantation is an effective treatment for patients with Type I diabetes mellitus. It leads to improved quality of life, independence from exogenous insulin administration, normalisation of glucose homeostasis, and can ameliorate diabetes-mediated organ dysfunction [8-12]. Despite continuing improvements in patient and allograft survival after SPK transplantation, surgical complications leading to organ loss or reoperation are common [1315]. This series examines the outcomes of Australia's largest whole-organ pancreas transplant service over the last decade.

A variety of techniques to manage pancreatic exocrine secretions have been described. These include enteric drainage, bladder drainage, duct occlusion, and open drainage into the peritoneal cavity. The practice in our unit is to use enteric drainage (ED) in preference to bladder drainage (BD). While BD avoids exposing the operative field to enteric flora and permits monitoring of amylase secretion, ED has been shown to be at least equivalent to bladder drainage with regard to patient and graft survival $[16,17]$. Moreover, $\mathrm{ED}$ is associated with a lower incidence of urinary tract infections and urological complications, avoids the metabolic derangement associated with bicarbonate loss, and has a lower incidence of anastomotic revision [18-21].

We also advocate the orientation of the head of the pancreas in a caudal direction towards the pelvis. This configuration facilitates ease of conversion to bladder drainage in the event of a pancreatic leak, rather than resorting to graft pancreatectomy.

Pancreatic leak, while infrequent, was the most common cause of return to theatre. Salvage operations were effective at preserving organ function; in only two of fifteen patients was the pancreas lost due to the consequences of a leak. Our rate

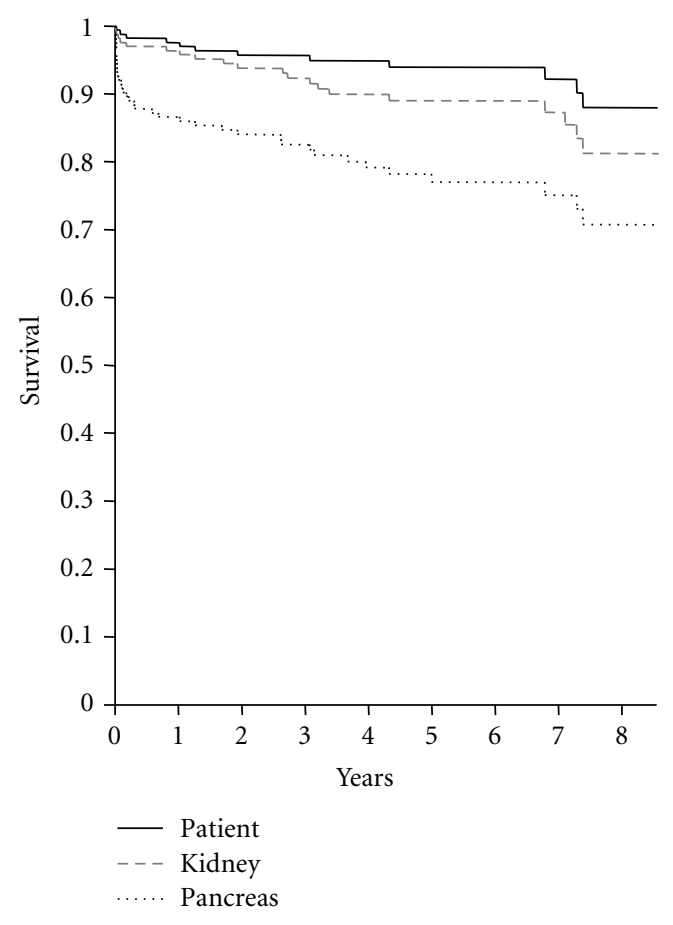

Figure 1: Patient and graft survival.

of allograft loss due to pancreatic leak (1.2\%) is comparable to those of large international centres. A $1.3 \%$ rate of pancreas loss secondary to leak in ED grafts was reported by UNOS [16]. Meanwhile, in a large single-centre experience, Sollinger et al. documented a leak rate of $5.7 \%$ in 610 enteric-drained pancreases, with the pancreas being lost in almost half of these [18].

While pancreatic leak is often amenable to re-intervention, pancreatic thrombosis remains the major challenge faced by transplant surgeons $[4,22]$. In this series, primary thrombosis accounted for 12 of 28 (50\%) death-censored graft failures and was responsible for $11 / 14$ pancreatic grafts (78.6\%) lost within a month of transplantation. Large series have documented thrombosis to be responsible for the majority of technical failures and early graft loss $[4,23]$. Factors that have been shown to predispose to graft thrombosis include recipient obesity, preservation time, and nontraumatic causes of donor death $[4,24]$.

Both medical and surgical strategies are used to minimise the risk of graft thrombosis. While we routinely use a short venous extension to the donor portal vein, it has been postulated that this may increase the risk of thrombosis [18]. Medical adjuncts to pancreas transplantation include the use of heparin or low-molecular-weight heparin [25], and commencing aspirin when oral intake begins. There is, however, little available evidence to support this in reducing graft thrombosis.

Our relaparotomy rates of $21.8 \%$ at one month and $34 \%$ overall are similar to those reported elsewhere $[14,15,18]$. Congruent with the recent findings of Manrique et al. [14], reoperation was associated with poorer graft, but not patient, survival. 
TABLE 4: Risk factors for pancreas graft loss at one year.

\begin{tabular}{|c|c|c|c|}
\hline & $\begin{array}{c}\text { Graft failure }<1 \text { year } \\
(n=23)\end{array}$ & $\begin{array}{l}\text { Functioning graft at } 1 \text { year } \\
\qquad(n=142)\end{array}$ & $P$ value \\
\hline Median donor age (years, IQR) & $33(21-39)$ & $26(20-36)$ & 0.17 \\
\hline Median donor BMI & $24.4(22.6-26.6)$ & $24.5(22.5-26.2)$ & 0.88 \\
\hline Median ratio of donor-to-recipient weight (IQR) & $1.10(0.94-1.41)$ & $1.08(0.93-1.29)$ & 0.87 \\
\hline Median recipient BMI (IQR) & $23.6(22.5-26.9)$ & $24.2(22.1-26.5)$ & 0.99 \\
\hline Nontraumatic donor death & $10(43.5 \%)$ & $44(31.0 \%)$ & 0.24 \\
\hline Prior dialysis $>1$ year & $18(78.3 \%)$ & $65(45.8 \%)$ & 0.03 \\
\hline Median pancreas anastomotic time (min, IQR) & $27(23-33)$ & $27(24-31)$ & 0.89 \\
\hline Median pancreas cold ischaemia time (hours, IQR) & $11.5(8.6-13.2)$ & $12.1(10.5-14.3)$ & 0.06 \\
\hline CMV-positive donor & $15(65.2 \%)$ & $94 / 138(68.1 \%)$ & 0.78 \\
\hline Return to theatre within 1 year & $17(74.0 \%)$ & $31(21.8 \%)$ & $<0.001$ \\
\hline
\end{tabular}

The five-year patient, pancreas, and kidney survival after SPK transplantation in this series are $94 \%, 77 \%$, and $89 \%$, respectively. This compares favourably with United States data in which five-year patient and pancreas-graft survival rates were $87 \%$ and $71-73 \%$, respectively, for patients who received a SPK transplant $[26,27]$.

There is evidence that SPK transplantation before the commencement of dialysis results in improved renal allograft outcomes [28]. Although we observed a trend towards improved kidney graft survival with preemptive transplantation, this did not reach statistical significance (log rank $P=0.07)$. Prior dialysis for more than one year, however, was associated with poorer one-year $(P=0.03)$ and longterm pancreas-graft survival (log-rank $P=0.02)$.

\section{Conclusion}

SPK transplantation has established benefits in improving quality of life and survival in Type 1 diabetic patients. Nevertheless it is an operation with well-defined morbidity, including that of pancreatic leak and thrombosis. In a country as large as Australia, our service faces the added logistical issues posed by recipients and donors being in distant locations. Despite this, we have demonstrated that SPK transplantation can be performed safely and successfully with functional and survival outcomes comparable to those achieved by large international centres.

\section{Conflict of Interests}

The authors declared that there is no Conflict of interests.

\section{References}

[1] W. D. Kelly, R. C. Lillehei, F. K. Merkel, Y. Idezuki, and F. G. Goetz, "Allotransplantation of the pancreas and duodenum along with the kidney in diabetic nephropathy," Surgery, vol. 61, no. 6, pp. 827-837, 1967.

[2] S. A. White, J. A. Shaw, and D. E. Sutherland, "Pancreas transplantation,” The Lancet, vol. 373, no. 9677, pp. 1808-1817, 2009.
[3] A. O. Ojo, H. U. Meier-Kriesche, J. A. Hanson et al., "The impact of simultaneous pancreas-kidney transplantation on long-term patient survival," Transplantation, vol. 71, no. 1, pp. 82-90, 2001.

[4] A. Humar, T. Ramcharan, R. Kandaswamy, R. W. G. Gruessner, A. C. Gruessner, and D. E. R. Sutherland, "Technical failures after pancreas transplants: why grafts fail and the risk factors-a multivariate analysis," Transplantation, vol. 78, no. 8, pp. 1188-1192, 2004.

[5] V. W. T. Lam, H. C. C. Pleass, W. Hawthorne, and R. D. M. Allen, "Evolution of pancreas transplant surgery," ANZ Journal of Surgery, vol. 80, no. 6, pp. 411-418, 2010.

[6] V. W. T. Lam, K. Wong, W. Hawthorne et al., "The linear cutting stapler for enteric anastomosis: a new technique in pancreas transplantation," Transplant International, vol. 19, no. 11, pp. 915-918, 2006.

[7] J. Hintze, NCSS, NCSS, LLC, Kaysville, Utah, USA, 2007.

[8] R. W. Bilous, S. M. Mauer, D. E. R. Sutherland, J. S. Najarian, F. C. Goetz, and M. W. Steffes, "The effects of pancreas transplantation on the glomerular structure of renal allografts in patients with insulin-dependent diabetes," New England Journal of Medicine, vol. 321, no. 2, pp. 80-85, 1989.

[9] P. Fioretto, M. W. Steffes, D. E. R. Sutherland, F. C. Goetz, and M. Mauer, "Reversal of lesions of diabetic nephropathy after pancreas transplantation," New England Journal of Medicine, vol. 339, no. 2, pp. 69-75, 1998.

[10] R. D. M. Allen, I. S. Al-Harbi, J. G. L. Morris et al., "Diabetic neuropathy after pancreas transplantation: determinants of recovery," Transplantation, vol. 63, no. 6, pp. 830-838, 1997.

[11] J. W. Jukema, Y. F. C. Smets, J. W. Van Der Pijl et al., "Impact of simultaneous pancreas and kidney transplantation on progression of coronary atherosclerosis in patients with end-stage renal failure due to type 1 diabetes," Diabetes Care, vol. 25, no. 5, pp. 906-911, 2002.

[12] A. O. Gaber, M. N. Wicks, D. K. Hathaway, and B. S. Burlew, "Sustained improvements in cardiac geometry and function following kidney-pancreas transplantation," Cell Transplantation, vol. 9, no. 6, pp. 913-918, 2000.

[13] C. Troppmann, "Complications after pancreas transplantation," Current Opinion in Organ Transplantation, vol. 15, no. 1, pp. 112-118, 2010.

[14] A. Manrique, C. Jiménez, R. M. López et al., "Relaparotomy after pancreas transplantation: causes and outcomes," Transplantation Proceedings, vol. 41, no. 6, pp. 2472-2474, 2009. 
[15] W. Steurer, J. Malaise, W. Mark, A. Koenigsrainer, and R. Margreiter, "Spectrum of surgical complications after simultaneous pancreas-kidney transplantation in a prospectively randomized study of two immunosuppressive protocols," Nephrology Dialysis Transplantation, vol. 20, supplement 2, pp. ii54-ii61, 2005.

[16] A. C. Gruessner and D. E. R. Sutherland, "Pancreas transplant outcomes for United States (US) and non- US cases as reported to the United Network for Organ Sharing (UNOS) and the International Prancreas Transplant Registry (IPTR) as of June 2004," Clinical Transplantation, vol. 19, no. 4, pp. 433-455, 2005.

[17] R. D. Bloom, M. Olivares, L. Rehman, R. M. Raja, S. Yang, and F. Badosa, "Long-term pancreas allograft outcome in simultaneous pancreas-kidney transplantation: a comparison of enteric and bladder drainage," Transplantation, vol. 64, no. 12, pp. 1689-1695, 1997.

[18] H. W. Sollinger, J. S. Odorico, Y. T. Becker, A. M. D’Alessandro, and J. D. Pirsch, "One thousand simultaneous pancreas-kidney transplants at a single center with 22-year follow-up," Annals of Surgery, vol. 250, no. 4, pp. 618-630, 2009.

[19] C. Jiménez-Romero, A. Manrique, J. C. Meneu et al., "Compative study of bladder versus enteric drainage in pancreas transplantation," Transplantation Proceedings, vol. 41, no. 6, pp. 2466-2468, 2009.

[20] D. P. Hickey, R. Bakthavatsalam, C. A. Bannon, K. O’Malley, J. Corr, and D. M. Little, "Urological complications of pancreatic transplantation," Journal of Urology, vol. 157, no. 6, pp. 2042-2048, 1997.

[21] M. Adamec, L. Janoušek, K. Lipár et al., "A prospective comparison of bladder versus enteric drainage in vascularized pancreas transplantation," Transplantation Proceedings, vol. 36, no. 4, pp. 1093-1094, 2004.

[22] H. W. Sollinger, "Pancreatic transplantation and vascular graft thrombosis," Journal of the American College of Surgeons, vol. 182, no. 4, pp. 362-363, 1996.

[23] F. L. Luan, M. Kommareddi, D. M. Cibrik, M. Samaniego, and A. O. Ojo, "Influence of recipient race on the outcome of simultaneous pancreas and kidney transplantation," American Journal of Transplantation, vol. 10, no. 9, pp. 2074-2081, 2010.

[24] M. S. Sampaio, P. N. Reddy, H. T. Kuo et al., "Obesity was associated with inferior outcomes in simultaneous pancreas kidney transplant," Transplantation, vol. 89, no. 9, pp. 1117-1125, 2010.

[25] P. Schenker, O. Vonend, N. Ertas et al., "Incidence of pancreas graft thrombosis using low-molecular-weight heparin," Clinical Transplantation, vol. 23, no. 3, pp. 407-414, 2009.

[26] L. Benetti, X. Bassi, G. Zamboni et al., "Pancreaticoduodenal graft in the rat: an original microsurgical technique," European Surgical Research, vol. 21, no. 3-4, pp. 162-167, 1989.

[27] K. Waki, P. I. Terasaki, and T. Kadowaki, "Long-term pancreas allograft survival in simultaneous pancreas-kidney transplantation by era: UNOS registry analysis," Diabetes Care, vol. 33, no. 8, pp. 1789-1791, 2010.

[28] A. K. Israni, H. I. Feldman, K. J. Propert, M. Leonard, and K. C. Mange, "Impact of simultaneous kidney-pancreas transplant and timing of transplant on kidney allograft survival," American Journal of Transplantation, vol. 5, no. 2, pp. 374-382, 2005. 


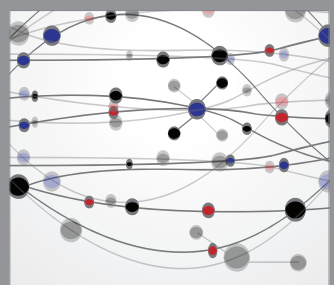

The Scientific World Journal
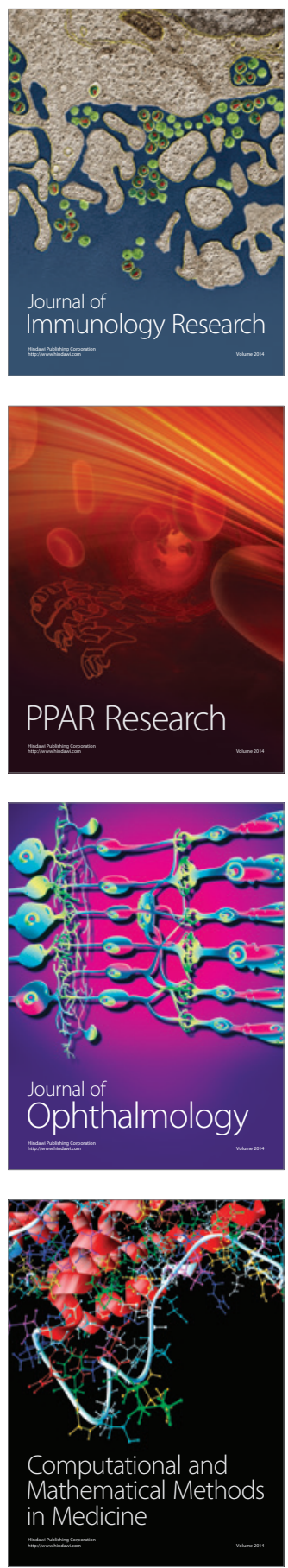

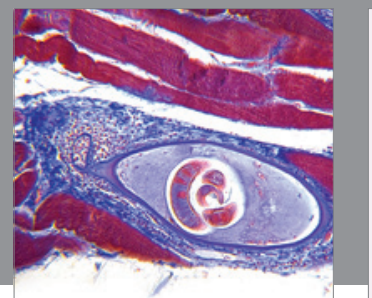

Gastroenterology

Research and Practice
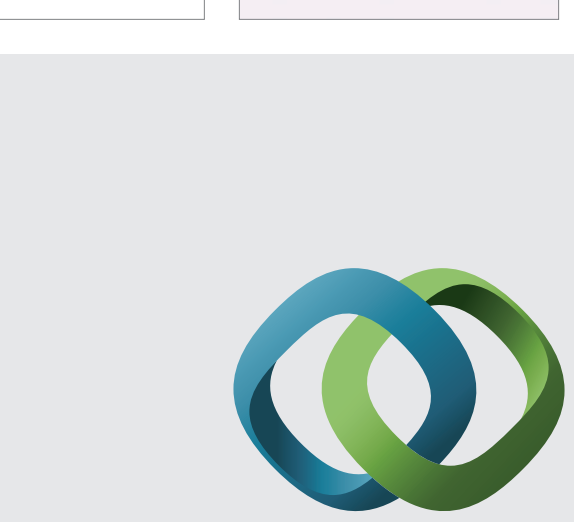

\section{Hindawi}

Submit your manuscripts at

http://www.hindawi.com
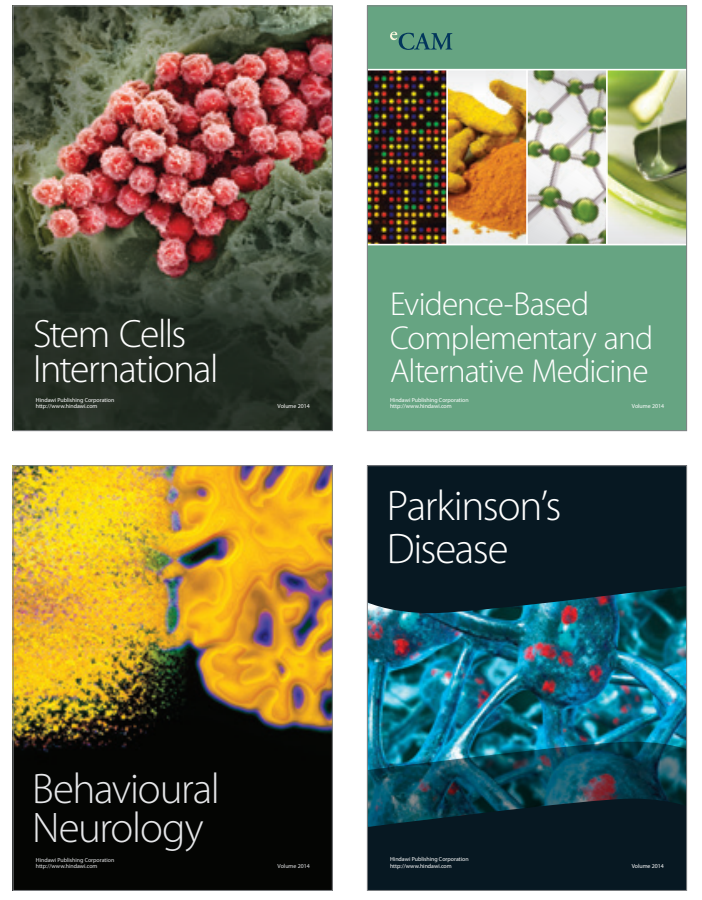
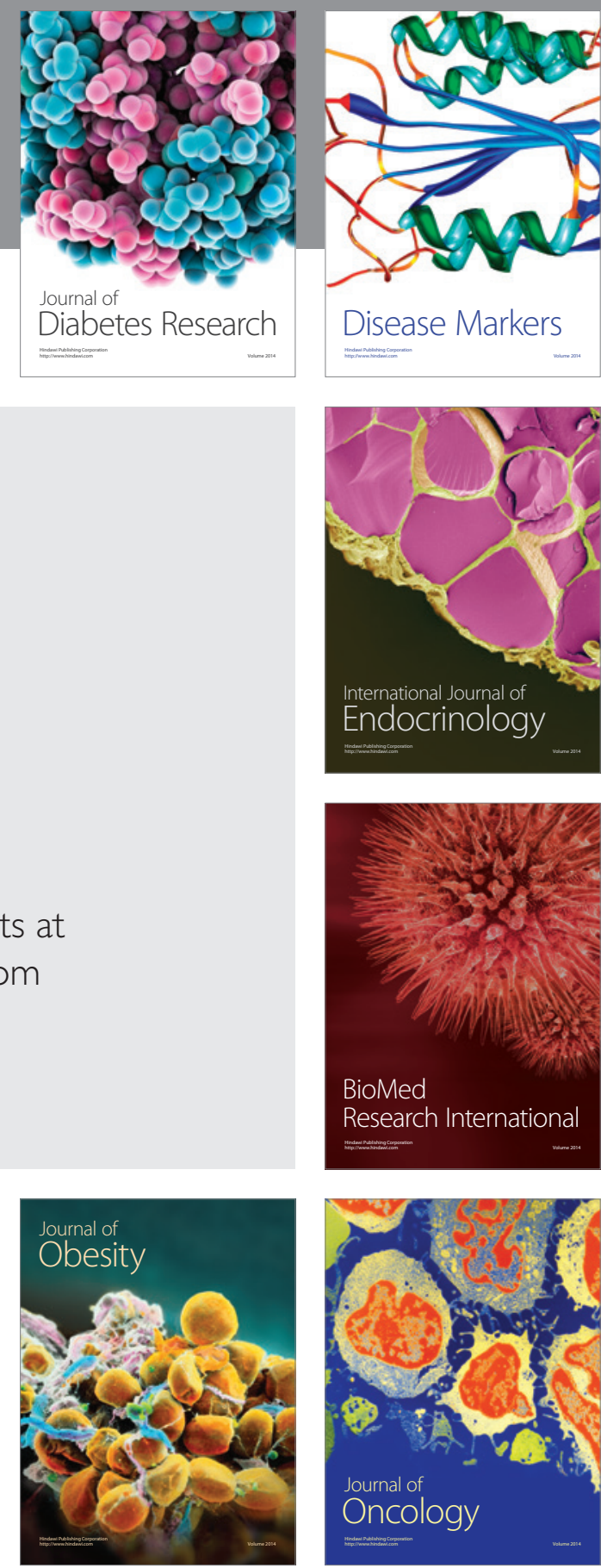

Disease Markers
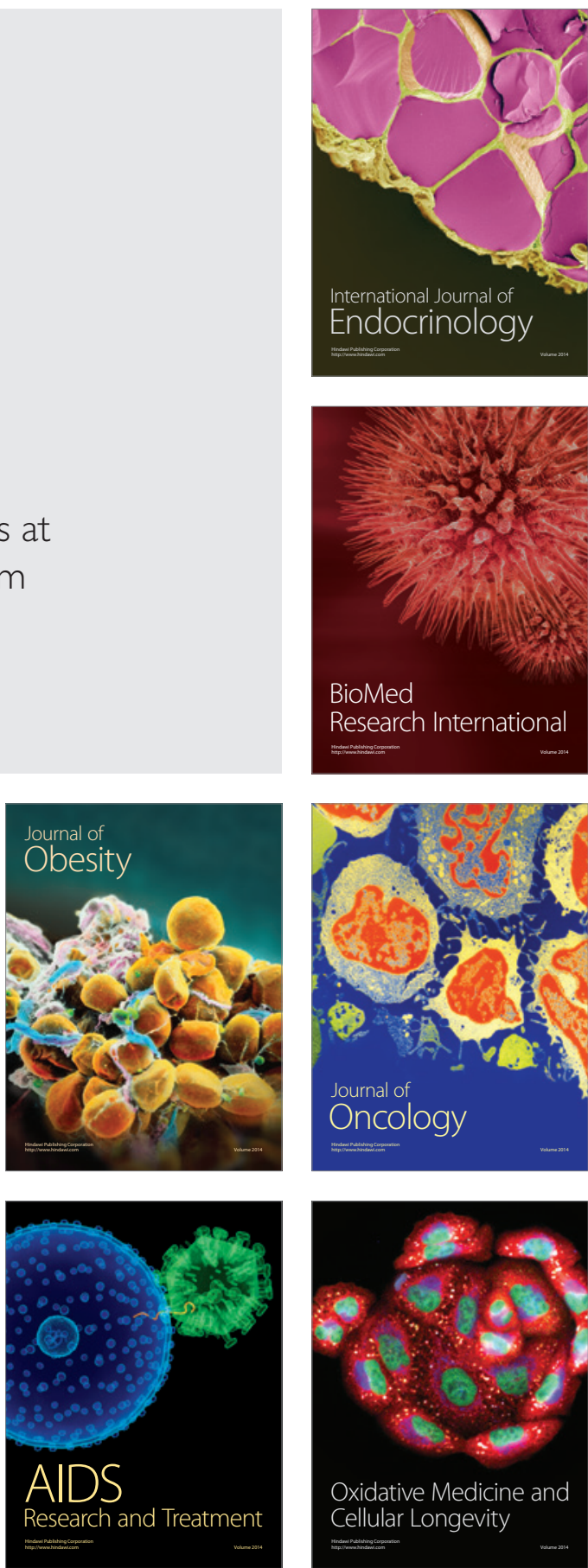